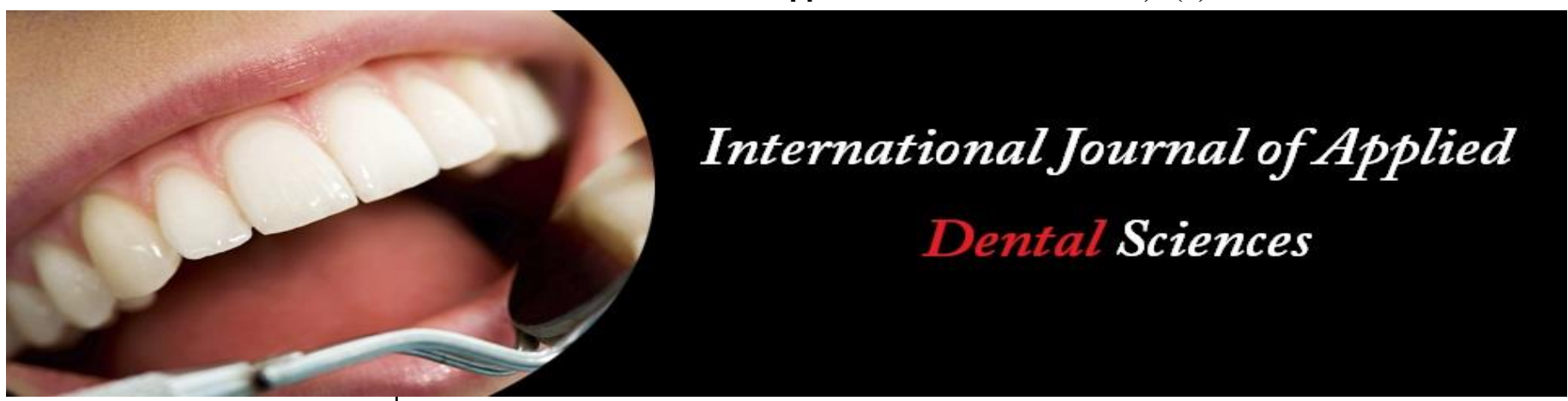

ISSN Print: 2394-7489

ISSN Online: 2394-7497

IJADS 2021; 7(1): 358-362

(C) 2021 IJADS

www.oraljournal.com

Received: 20-11-2020

Accepted: 24-12-2020

Manar Motawie Abdulfattah Faculty of Dentistry, Cairo

University, Cairo, Egypt

Sara Ahmed Mahmoud

Pediatric Dentistry and Dental

Public Health Department,

Faculty of Dentistry, Cairo

University, Cairo, Egypt

Soad Abdel moniem Abdel moniem

Pediatric Dentistry and Dental Public Health Department, Faculty of Dentistry, Cairo University, Cairo, Egypt
Corresponding Author: Manar Motawie Abdulfattah Faculty of Dentistry, Cairo University, Cairo, Egypt

\section{Post-operative pain after $38 \%$ silver diamine fluoride application in primary molars with deep caries versus interim restorative therapy: A randomized pilot study}

\author{
Manar Motawie Abdulfattah, Sara Ahmed Mahmoud and Soad Abdel \\ moniem Abdel moniem
}

DOI: https://doi.org/10.22271/oral.2021.v7.i1e.1155

\section{Abstract}

Aim: To compare the post-operative pain after application of Silver Diamine Fluoride (SDF) 38\% in cavitated primary molars, in relation to Interim Restorative Therapy (IRT).

Design: The study was performed on 20 carious primary molars in children aged 4-6 years attending the clinic in Pediatric Dentistry and Dental Public Health Department- Faculty of Dentistry -Cairo University. Partial excavation of caries on the walls of the cavity was performed then in group (A) SDF was applied in two visits with one-week interval, and finally Resin Modified Glass Ionomer Restoration (RMGI) was applied to the cavity. While, in group (B) Resin Modified Glass Ionomer restoration was applied immediately after partial caries excavation. Clinical and radiographic examinations were performed at three and six month's follow-up periods for both groups.

Results: In both groups, $100 \%$ of patients had no complications at three and six months follow up periods; except for $10 \%$ of patients experienced failure of restoration at three months in group B. The results showed insignificant differences between groups regarding clinical and radiographic outcomes at both three and six months.

Conclusions: Silver Diamine Fluoride and Interim Restorative Therapy were clinically and radiographically successful when used in primary molars with cavitated dentin carious lesions.

Keywords: Pain, primary molars, interim restorative therapy, silver diamine fluoride

\section{Introduction}

Dental caries is a chronic disease which presents a major problem for the individual and the population. Untreated dental caries in deciduous teeth was the tenth-most prevalent condition, affecting $9 \%$ of the global population. When caries is left untreated, pulp vitality may be jeopardized leading to pain, infection, swelling and premature loss of deciduous teeth which leads to functional and esthetic problems ${ }^{[1]}$.

Complete removal of dental caries has been the treatment of choice for many decades. However, this technique showed many disadvantages as the removal of a significant quantity of dental structure which weakens the tooth, and the elevated risk of pulp tissue exposure. Moreover, performing this technique for caries removal and teeth restoration in pediatric patients may be difficult, especially for young or apprehensive children, who cannot cooperate during the dental visit ${ }^{[2]}$.

Arresting Caries Treatment is a minimally invasive approach which is based on preventive treatment to stop the progression of the disease, preserving the vitality of the pulp through remineralization and minimal intervention in the dental tissue. Atraumatic Restorative Treatment, Interim Restorative Therapy (IRT) and Silver Diamine Fluoride (SDF) were introduced to serve the approach of sealing in and arresting dental caries ${ }^{[3]}$.

According to (dos Santos et al., 2012) ${ }^{[4]}$, who conducted a randomized controlled study to compare the effect of IRT (FUJI IX) with 30\% SDF in 91 children aged 5-6 years, SDF application showed to be more effective, after 1 year, than IRT in caries arrest. Moreover, (Zhi et al., 2012) ${ }^{[5]}$ performed a study on 212 children, of age 3-4 years to compare the effect of (1) annual application of SDF, (2) semi-annual application of SDF, and (3) annual application of 
glass ionomer on carious dentin cavities in primary teeth and it was concluded that there was no significant difference between the effectiveness of the annual application of SDF solution and that of annual application of glass ionomer in arresting active dentin caries in primary teeth and that increasing the frequency of application of SDF solution to every 6 months can increase the caries arrest rate.

Since only few high quality studies on whether to use SDF or IRT have been published, thus, the aim of this study was to evaluate the clinical and radiographic success of SDF and IRT in primary molars with cavitated dentin carious lesions.

\section{Methods}

This study was a randomized pilot clinical trial with 1:1 parallel groups. The ethical clearance for this study was obtained from the Committee of Ethics in Faculty of Dentistry, Cairo University. This trial has been registered with clinicaltrials.gov under the title: Treatment of Primary Molars with Deep Caries Using Silver Diamine Fluoride with registration number NCT03563534. Sample size calculation was not required as this intervention is new, therefore, a pilot study was recommended. The suggested total sample size was 20 (ten molars per group).

A total of 20 primary molars in eleven patients were selected randomly from the outpatient clinic of Pediatric Dentistry and Dental Public Health Department, Faculty of Dentistry, Cairo University and a written consent was obtained from all legal guardians. Apparently medically free children aged 4-6 years, with no history of pain with exception of sensitivity while eating or drinking, were recruited. Clinical examination was performed using a mirror and sharp explorer to assess inclusion criteria, and radiographic examination was done using preoperative periapical X-ray films size 0 through an Xray machine, radiographic stents and extension cone paralleling (XCP) film holder. The molars included in the study had dentin carious lesions on the occlusal surface corresponding to International Caries Detection and Assessment System (ICDAS) codes 5 or 6 (distinct or extensive cavity with visible dentine), normal appearance of the gingiva (absence of swelling/sinus or fistula) and absence of peri-apical or inter-radicular radiolucency, widening of periodontal ligament space, and internal or external root resorption. Sequence generation was done for the patient number (1-20) using computer sequence generation (www.random.org) and the assistant supervisor informed the operator to place the tooth in which group according to the random table and the patient assignment was done by the operator. Afterwards, children were randomly allocated with 1:1 allocation ratio into two groups $(n=10)$ based on the material used. Group A: Silver Diamine Fluoride (SDF), Group B: Interim Restorative Therapy (IRT).

The carious teeth were isolated using cotton rolls and partial excavation was performed for caries around the margins of the cavity only without removal of caries at the floor of the cavity using excavator. In Group A (SDF), Scented lip balm was applied to the patient's lips and the patient was instructed to wear protective eyewear, then a micro brush was dipped in a drop of SDF (Advantage Arrest, Elevate Oral Care LLC) and was placed on the lesion(s) for one minute and the excess material was removed using a cotton roll. The patient then was dismissed and was instructed to come again after one week for a second application. In the second visit, after cleaning the cavity and the removal of any food debris, a second application of SDF was done for one minute, and then rinsing with water. After that, the cavity was dried and Resin Modified Glass Ionomer restoration (EQUIA FIL capsule, GC America) was applied and the patient was instructed not to eat or drink for one hour after application in both visits. In Group B (IRT), Resin Modified Glass Ionomer restoration (EQUIA FIL capsule, GC America) was applied to the cavitated dentin carious lesions immediately after marginal caries excavation and the patient was instructed not to eat or drink for one hour after application. In both groups, immediate post-operative photograph was taken and peri-apical radiograph was performed using the radiographic stent and the XCP film holder and children were instructed to brush their teeth at least two times per day using the same standardized technique (Bass technique). The teeth brushing technique was educated by the operator, and the children practiced it before being dismissed.

Primary outcome was history of pain using direct questioning at three and six months follow-up periods, secondary outcomes were 1) absence of swelling, sinus or fistula using visual examination at three and six months follow-up period, 2) absence of pain on percussion using percussion test at three and six months follow-up period, 3) radiographic success (defined as the absence of inter-radicular or periapical radiolucency, widening of PDL space, internal or external root resorption) using peri-apical radiograph parallel technique at three and six months follow-up period, 4) failure of restoration (marginal leakage, recurrent caries, loss of the restoration) using visual and tactile examination at three and six months follow-up period and 5) parent satisfaction by a questionnaire using the Visual Analogue Scale on a scale from (0-10) at six months follow-up period, the assistant supervisor gave the parents the questionnaire to assess their satisfaction using the Visual Analogue Scale (VAS) without knowing the patient group to avoid bias.

The operator was semi-blinded (partial excavation of caries was performed and finished without knowing which material will be used). The radiographic evaluator and the statistician were blind to whether it is an experimental or a control group.

\section{Statistical Analysis}

Numerical variables (Parental satisfaction on VAS scale) were described in terms of mean, median, standard deviation (SD) and range according to each group. Categorical binary variables (Clinical and radiographic records) were described in terms of frequencies and percentages. The significance level was set at $P \leq 0.05$. The statistical package used for this study was R statistical package, version 3.5.2 (20-12-2018).

\section{Results}

20 primary molars were divided equally into two groups, group A: SDF and group B: IRT. Cases were evaluated according to the clinical and radiographic criteria at three and six months with no drop out of patients at the end of the study.

\section{Clinical and Radiographic outcomes a) Intra-group comparison}

In SDF group, 10 patients $(100 \%)$ had no complications at 3 and 6 months follow-ups. The results showed statistically insignificant changes from 3 to 6 months. In IRT group, 10 patients $(100 \%)$ had no complications at 3 and 6 months follow-ups; only 1 patient (10\%) experienced failure of restoration at 3 months. The results showed statistically insignificant changes from 3 to 6 months. 


\section{b) Inter-group comparison}

The results showed insignificant differences between groups regarding clinical and radiographic outcomes at both 3 and 6 months. There was no pain, swelling/fistula/sinus, pain on percussion, inter-radicular/periapical radiolucency, widening of periodontal membrane space, or internal/external resorption in both groups at 3 and 6 months follow-up periods.

\section{Parental satisfaction assessment}

The Mean Visual Analogue Scale (VAS) scores for the simplicity of the procedure, the time needed for the procedure, and the attitude of the child toward the treatment for SDF and IRT groups were identical $(10 \pm 0)$; i.e. there was no difference between groups regarding the previously mentioned points. The Mean VAS scores for the performance of the restoration for SDF Group was $10( \pm 0)$; while the mean score for IRT Group was $9.5( \pm 1.58)$; i.e. the difference between the two groups was statistically insignificant. While the Mean VAS scores for Esthetics for SDF Group was 7.6 $( \pm 2.07)$; and IRT Group was $10( \pm 0)$; i.e. the difference between the two groups was statistically significant (IRT Group was more satisfactory related to esthetics than SDF Group).

\section{Discussion}

Many studies have been published on Silver Diamine Fluoride and Interim Restorative Treatment and both were considered successful minimally invasive treatments in arresting dental caries.

The chosen study design was Randomized Pilot Study because the assessment of the feasibility is the main goal of pilot studies.

In the present study, the eligibility criteria were determined by guidelines of (AAPD, 2017a) ${ }^{[6]}$ because if the included molars were treated by the conventional operative procedures (high-speed hand-piece and complete removal of caries), they would require pulp therapy with a suitable restoration.

In January 2019, the Committee for Protection of Human Subjects published guidelines for children in research which recommended that children up to seven years old are not able to participate in oral assent process and so, only informed consents were taken from the parents ${ }^{[7]}$.

Preoperative periapical radiographs were performed for the primary molars to confirm the adherence of the participant to the inclusion criteria. Radiographic examination was conducted using intraoral periapical radiograph (parallel technique) as it is considered the most reliable method for detection and long term assessment of periapical and furcation pathologies ${ }^{[8]}$. The parallel technique was the technique of choice for radiographic evaluation to obtain accurate and undistorted images using film holders. Also, radiographic stents were used to ensure reproducibility.

Conventional radiography was used in this study as it provides easy and comfortable radiograph to young children [9]. The assessment of the radiographs at the follow-up periods was done by a blinded assessor not involved in treating the teeth or supervising the treatment to avoid bias.

The isolation technique used in this study was three cotton rolls as it is simple and easy to be used in procedures like sealant and topical fluoride application and also because local anesthesia was not given to the patients so placement of clamps and rubber dam was not applicable ${ }^{[10]}$.

Marginal caries excavation was performed in both groups (RMGI restorations were used in both groups) as this helps in placing the peripheral Resin Modified Glass Ionomer (RMGI) restoration onto sound dentin for better adhesion and to achieve an adequate seal that minimizes leakage and inactivate remaining bacteria. In addition, marginal caries excavation maintains pulpal health by leaving soft caries in proximity to the pulp to avoid unwanted irritation or pulp exposure ${ }^{[11]}$. In Group A (SDF Group), marginal caries excavation may have an added advantage which is minimizing the amount of arrested caries that changes black and so gives better esthetics ${ }^{[6]}$.

Vaseline was applied on the gingival margin and lips, in Group A (SDF), to protect the gingiva and the lips from pigmentation and avoid soft tissue irritation. Also, protective eyewear was used in the SDF Group, for more protection for the child's eye ${ }^{[12]}$.

In Group A, 38\% SDF was used as studies have shown that it is more effective in arresting caries than $12 \%$ SDF especially if applied twice a year ${ }^{[13]}$. The application time was one minute which gives enough time for the absorption of SDF and the excess material was removed with cotton to minimize the systemic absorption of SDF ${ }^{[6]}$.

In Group B, the IRT approach was used as according to Massara et al. ${ }^{[14]}$ it helps in creating favorable conditions for the healing process of remaining demineralized dentin in primary molars with deep caries. Moreover, Hof et al. [15] concluded that high survival rates for single-surface restorations were found in both primary and permanent dentitions using high-viscosity glass-ionomers. In addition, Singhal et al. ${ }^{[16]}$ concluded that partial caries removal using IRT was found to be an effective procedure for caries management.

The Visual Analogue Scale was used to measure parent satisfaction as the items of the Visual Analogue Scale were less vulnerable to bias from confounding factors and needed shorter time to complete the questionnaire ${ }^{[17]}$.

Regarding the present study, the statistician was completely blinded to the treatment groups. Complete blinding of the operator, children and their parents was not possible due to the visual appearance of both study materials (Silver Diamine Fluoride was liquid while Interim Restorative Therapy was Glass Ionomer paste). Nevertheless, to overcome this problem, the operator was (semi-blinded) to the material (the operator didn't know which material was going to be used) from the beginning of treatment till the end of the excavation of caries, which is a critical step and might bias the results.

Regarding the results of the study, there was no statistical difference in the primary outcome (clinical assessment) between the Silver Diamine Fluoride group (group A) and the Interim Restorative Therapy group (group B). All involved teeth were free of spontaneous post-operative pain, swelling/fistula or sinus and pain on percussion at $3 \& 6$ months follow-up intervals.

In the current study, no statistical difference was found in the secondary outcome which was radiographic evaluation, between the Silver Diamine Fluoride group and the Interim Restorative Therapy group. All involved teeth were free of periapical/inter-radicular radiolucency, widening of periodontal membrane space and internal/external resorption at $3 \& 6$ months follow-up periods.

The successful effect of SDF in arresting the remaining dentin caries was due to its mechanism of action on dental caries which is 1) inhibition of demineralization and allowing remineralization, 2) blocking the dentinal tubules, 3) the antienzymatic effect against the cariogenic bacteria ${ }^{[18]}$. It has also been proposed that the fluoride ion can affect the physiology 
of microbial cells, including cariogenic streptococci, which can thus indirectly affect demineralization ${ }^{[19]}$.

The reaction of metallic silver (which is inert) with the moisture in the oral environment leads to the production of silver ions (Ag+) which have three actions against bacteria 1) destruction of the cell wall structure, 2) denaturation of cytoplasmic enzyme, and 3) inhibition of bacterial DNA replication ${ }^{[20]}$.

The clinical and radiographic success of the IRT in arresting caries may occur due to the use of RMGI restoration, which exhibits effective antimicrobial activity against cariogenic bacteria. This activity is attributed to two reasons which are: 1) the low initial $\mathrm{pH}, 2$ ) the release of chemical components such as fluoride ${ }^{[21]}$.

The results of the clinical \&radiographic outcomes of the current study were in accordance to Zhi et al. ${ }^{[5]}$ who stated that both Silver Diamine Fluoride \& Interim Restorative Therapy were successful in arresting caries in primary teeth with cavitated dentin carious lesions.

A sign of clinical failure was reported in $(10 \%)$ of the Resin Modified Glass Ionomer Restorations of the Interim Restorative Therapy Group at 3 months follow-up. This type of failure was in the form of complete dislodgment (loss) of the RMGI restoration which may be related to the patient dietary habits (eating sticky food). The child complained only from sensitivity while eating and drinking, and after clinical examination the floor of the cavity was hard, the cavity was cleaned and RMGI restoration was applied again. At 6 months follow-up, no failure of the restoration, and the patient had no complaints.

The results of the parent satisfaction questionnaire using the Visual Analogue Scale showed no statistical difference between both groups in relation to the simplicity of the procedure which supports the findings of Clemens et al. ${ }^{[22]}$. Moreover, there was no statistical difference found between both groups in relation to time needed for the procedure, attitude of the child toward the treatment and performance of the restoration. These findings were probably because both approaches are simple, easy, not requiring a lot of time, accepted by young children and help the child to adapt and become familiar with the dental treatment (buying time for the child) without the need for General Anesthesia.

However, the results of parent satisfaction in relation to esthetics were statistically significant, where Group B (IRT) was more satisfactory than Group A (SDF), the same findings were reported by Alshammari et al. ${ }^{[23]}$ and Bagher et al. ${ }^{[24]}$. Parents' acceptance of the Interim Restorative Therapy was $100 \%$ where all of them gave it score 10 (totally satisfied) on the Visual Analogue Scale, while parents' acceptance of the Silver Diamine Fluoride was variable. This can be explained by the high esthetic demand of the patients and their parents where children even as young as three years were aware of their appearance ${ }^{[25]}$.

Treatment of dentin carious molars with these different treatment modalities (SDF \& IRT) reported clinical and radiographic success in addition to the cost of both treatments which is considered low and affordable for most of the patients in comparison to other alternative treatments as pulp therapy/crowns or treatment under General Anesthesia. This provides a shift of dental caries management protocol towards the minimally invasive dentistry with promising long term success.

\section{Conclusion}

Silver Diamine Fluoride and Interim Restorative Therapy showed clinical and radiographic success when used with partial caries removal in primary molars with cavitated dentin carious lesions. Parents were more satisfied with the esthetics of Interim Restorative Therapy than Silver Diamine Fluoride.

\section{Why this paper is important to pediatric dentists}

- Both Silver Diamine Fluoride and Interim Restorative Therapy are minimally invasive approaches that provide an easy, time saving, and cost-effective way of treatment especially in pediatric dentistry.

- Silver Diamine Fluoride and Interim Restorative Therapy are very helpful and effective in young, uncooperative, or handicapped children who cannot be managed by other long restorative procedures.

- $\quad$ Silver Diamine Fluoride and Interim Restorative Therapy can be performed in remote and underprivileged areas where no dental facilities are available.

- Silver Diamine Fluoride and Interim Restorative Therapy can reduce the need for General Anesthesia which takes more time, effort and cost.

\section{References}

1. Al-shahrani N, Al-amri A, Hegazi F, Al-rowis K, Almadani A, Hassan K. The prevalence of premature loss of primary teeth and its impact on malocclusion in the Eastern Province of Saudi Arabia. Acta Odontologica Scandinavica 2015;73(7):544-549. https://doi: 10.3109/00016357.2014.939709.

2. Fung MHT, Wong MCM, Lo ECM, Chu CH. 'Arresting Early Childhood Caries with Silver Diamine Fluoride- A Literature Review. Journal of Oral Hygiene \& Health 2013;1(3):1-5. 0702.1000117 .

https://doi.org/10.4172/2332-

3. Neena IE, Edagunji G, Poornima P, Nagaveni NB, Roopa KB, Bharath KP. Minimal invasive dentistry. International Journal of Contemporary Dental and Medical Reviews, 2015, 1-4. https://doi: 10.15713/ins.ijcdmr.40.

4. dos Santos VE, de Vasconeclos FMN, Ribeiro AG, Rosenblatt A. Paradigm shift in the effective treatment of caries in schoolchildren at risk. International Dental Journal 2012;62:47-51. https://doi.org/10.1111/j.1875595X.2011.00088.X.

5. Zhi QH, Man lo EC, Lin HC. Randomized clinical trial on effectiveness of silver diamine fluoride and glass ionomer in arresting dentine caries in preschool children. Journal of Dentistry 2012;40(11):962-967. https://doi.org/10.1016/j.jdent.2012.08.002.

6. AAPD. Use of Silver Diamine Fluoride for Dental Caries Management in Children and Adolescents, Including Those with Special Health Care Needs. Pediatric Dentistry 2017;40(6):152-161.

7. Committee for Protection of Human Subjects (CPHS) Child assent and parent permission, 2019, 1-7.

8. Kumar V, Arora K, Udupa H. Different Radiographic Modalities Used for Detection of Common Periodontal and Periapical Lesions Encountered in Routine Dental Practice. Journal of Oral Hygiene \& Health, 2014;2(5):111. https://doi: 10.4172/2332-0702.1000163.

9. Davies A, Mannocci F, Mitchell P, Andiappan M, Patel, $\mathrm{S}$. The detection of periapical pathoses in root filled teeth using single and parallax periapical radiographs versus cone beam computed tomography - a clinical study. International Endodontic Journal 2015;48(6):582-592. https://doi.org/10.1111/iej.12352. 
10. Gautam A, Srivastava N, Rana V, Kaushik N. Isolation in pediatric dentistry. European Journal of Pharmaceutical and Medical Research 2019;6(5)491-495.

11. Schwendicke F, Frencken JE, Bjørndal L, Maltz M, Manton DJ, Ricketts D, et al. Managing Carious Lesions: Consensus Recommendations on Carious Tissue Remova. Advances in dental research 2016;28(2):58-67. https://doi: 10.1177/0022034516639271.

12. Horst JA, Ellenikiotis H. UCSF Silver Caries Arrest Committee, Milgrom PM. 'UCSF Protocol for Caries Arrest Using Silver Diamine Fluoride: Rationale, Indications, and Consent'. California Dental Assossiation Journal 2016;44(1):16-28.

13. Fung MHT, Duangthip D, Wong MCM, Lo ECM, Chu CH. Arresting Dentine Caries with Different Concentration and Periodicity of Silver Diamine Fluoride. Journal of Oral Hygiene \& Health 2016;1(2):143-152. https://doi.org/10.1177/2380084416649150.

14. Massara MLA, Alves JB, Brandão PRG. Atraumatic restorative treatment: Clinical, ultrastructural and chemical analysis. Caries Research, 2002;36:430-436. https://doi.org/10.1159/000066534.

15. Hof MA, Frencken JE, Helderman W, Holmgren CJ. The Atraumatic Restorative Treatment (ART) approach for managing dental caries: a meta-analysis. International Dental Journal 2006;56:345-35.

16. Singhal DK, Acharya S, Thakur AS. Microbiological analysis after complete or partial removal of carious dentin using two different techniques in primary teeth : A randomized clinical trial. Dental Research Journal 2016;13(1):30-37.

17. Voutilainen A, Pitk €Aaho T, Kvist T, Julkunen KV. 'How to ask about patient satisfaction? The visual analogue scale is less vulnerable to confounding factors and ceiling effect than a symmetric Likert scale. Journal of Advanced Nursing 2016;72(4):946-957. https://doi: 10.1111/jan.12875.

18. Shounia T, Atwan S, Alabduljabbar R. Using Silver Diamine Fluoride to Arrest Dental Caries: A New Approach in the US. Journal of Dentistry and Oral Biology 2017;2(18):22-25.

19. Liao Y, Brandt BW, Li J, Crielaard W, Loveren CV, Deng DM. Fluoride resistance in Streptococcus mutans: a mini review. Journal of Oral Microbiology 2017;9(1):19. https://doi: 10.1080/20002297.2017.1344509.

20. Kędziora A, Speruda M, Krzyzewska E, Rybka J, Łukowiak A, Płoskonaska GB. Similarities and Differences between Silver Ions and Silver in Nanoforms as Antibacterial Agents. International Journal of Molecular Science 2018;19(444):1-17. https://doi: 10.3390/ijms19020444.

21. Lacet G, Ferreira S, Freires IDA, Alves LA, Jovito VDC. Antibacterial Activity of Glass Ionomer Cements on Cariogenic Bacteria - An in vitro study. International Journal of Dental Clinics 2011;3(3):1-3.

22. Clemens J, Gold J, Chaffin J. Effect and acceptance of silver diamine fluoride treatment on dental caries in primary teeth. Journal of Public Health Dentistry 2017;(7):1-6. https://doi.org/10.1111/jphd.12241.

23. Alshammari AF, Almuqrin AA, Aldakhil AM, Alshammari BH, Lopez J. Parental perceptions and acceptance of silver diamine fluoride treatment in Kingdom of Saudi Arabia. International Journal of Health Sciences 2019;13(2):25-29.
24. Bagher SM, Sabbagh HJ, Aljohani G, Aldajani M, Elkhodary H. Parental acceptance of the utilization of silver diamine fluoride on their child's primary and permanent teeth. Patient Preference and Adherence 2019;13:829-835.

25. Tarang C, Gunjan Y, Mani TA, Kavita D, Arora D. 'Recent trends of Esthetics in Pediatric Dentistry. International Journal of Oral Health and Medical Research 2017;4(4):70-75. 